- Case Report

\title{
Gentleman with the Purple Urine
}

\author{
Chong Yau Ong ${ }^{1,2, *}$, Farhad Fakhrudin Vasanwala ${ }^{1,2,3}$ \\ 'Department of Family Medicine, Sengkang Hospital, Singapore \\ ${ }^{2}$ Department of General Psychiatry 2, Institute of Mental Health, Singapore \\ ${ }^{3}$ SingHealth Duke-NUS Family Medicine Academic Clinical Program, Singapore
}

\begin{abstract}
A 50-year-old man was found to have purple discoloration of the urine. He was on long-term urinary catheterization. He was asymptomatic, and urinalysis did not suggest a urinary tract infection. Purple urine bag syndrome resolved with a change of the urinary catheter and bag. He was prescribed regular laxatives and passed stools regularly.
\end{abstract}

Keywords: Purple; Urine; Urinary Catheterization; Urinary Tract Infections

Received: June 5, 2018, Revised: July 11, 2018, Accepted: July 12, 2018

*Corresponding Author: Chong Yau Ong https://orcid.org/0000-0002-6230-7134

Tel: +65-81268091, E-mail: ong.chong.yau@singhealth.com.sg 


\section{INTRODUCTION}

Purple urine bag syndrome (PUBS), while widely reported in the literature, is still an under-recognized condition in health care facilities. It can be alarming and causes anxiety to patients, family members, and health care staff. Therefore, an understanding of this condition is paramount. We hereby present the case of a man with PUBS.

\section{CASE REPORT}

A 50-year-old man, a resident of a long-term care unit, was reviewed for diabetes care. The nurse in charge had particularly highlighted that there was a change in the color of his urine for 3 days (Figure 1).

He had a medical history of diabetes and subclinical hypothyroidism. He also had a background of benign prostatic hyperplasia and neurogenic bladder, requiring a long-term indwelling urinary catheter.

On examination, he was afebrile, without sepsis, and his hydration status was normal. There was no palpable bladder, and the renal punch was negative. Examination of the urine bag showed purple discoloration of the urine. He had no symptoms of urinary tract infection.

He was diagnosed to have PUBS. He had a history of urinary tract infections (Providencia rettgeri, June 2016; and Klebsiella pneumoniae, July 2016), which were successfully treated.

Urinary fluid and microscopy examination were not suggestive of a urinary tract infection. Therefore, he was not prescribed antibiotics. Although the catheter was not due for change, the urinary catheter and bag were changed. He was prescribed regular laxatives and asked to be potted regularly. The discoloration of urine resolved with a new urinary catheter and bag.

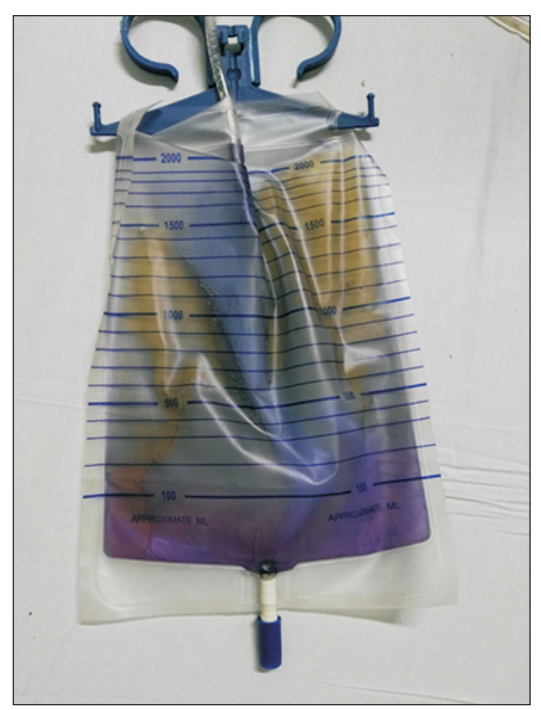

Figure 1. The purple discoloration of the urine and urine bag.

\section{DISCUSSION}

PUBS, although being relatively rare in clinical practice, has been well described in the literature. It was first described in 1978 with an estimated prevalence of $8.3 \%-42.1 \%$ among patients with long-term indwelling catheters. ${ }^{1)}$ Yang and $\mathrm{Su}^{2)}$ found that $58.3 \%$ of reported PUBS cases occurred in long-term care units.

The purple discoloration is caused by a combination of indigo (blueviolet) and indirubin (red-violet) pigments. Normal bacteria flora deaminates ingested tryptophan to produce indole. Indole is transported into the portal circulation and conjugated in the liver to produce indoxyl sulphate which is then catalyzed by sulfatases and phosphatases to form indoxyl. In alkaline urine (high $\mathrm{pH}$ ), indoxyl is oxidized to indigo and indirubin. ${ }^{3)}$ Interaction between these pigments and the urine tubing and bag causes the urine and bag to turn purple.

It is associated with organisms that have indoxyl phosphatase/sulphatase activity such as Klebsiella pneumoniae, Providentia stuartii, Enterobacter spp., Proteus mirabilis, Morganella morganii, and Escherichia coli. ${ }^{4)}$

Increased dietary tryptophan, alkaline urine, constipation, chronic catheterization, high urinary bacterial load, renal failure, and use of polyvinylchloride plastic catheters are among the identified risk factors for PUBS. ${ }^{5)}$ Decreased gut motility in conditions such as ileus, diverticular disease, and obstruction also predisposes to PUBS as the gut flora have more time to deaminate tryptophan. Constipation can cause urinary retention, therefore allowing bacteria in the urine more time to catalyze indoxyl sulphate. Renal failure leads to decreased clearance of indoxyl sulphate.

PUBS has not been proven to have any other implications other than the possibility of urinary tract infection, neither has it been shown to impact clinical outcomes of patients. ${ }^{6)}$ It is accepted that PUBS largely has a benign course and therefore, extensive investigations and treatment are not usually necessary. ${ }^{7)}$ Maintenance of bowel continence, good urinary catheter care, and treatment of confirmed urinary tract infections are highly recommended.

Physicians managing patients with long-term indwelling catheterization within chronic care institutions need to be aware of this condition.

\section{CONFLICT OF INTEREST}

No potential conflict of interest relevant to this article was reported.

\section{ORCID}

Chong Yau Ong: https://orcid.org/0000-0002-6230-7134

Farhad Fakhrudin Vasanwala: https://orcid.org/0000-0001-5749-9795

\section{REFERENCES}

1. Barlow GB, Dickson JA. Purple urine bags. Lancet 1978;311:220-1. 
2. Yang HW, Su YJ. Trends in the epidemiology of purple urine bag syndrome: a systematic review. Biomed Rep 2018;8:249-56.

3. Dealler SF, Hawkey PM, Millar MR. Enzymatic degradation of urinary indoxyl sulfate by Providencia stuartii and Klebsiella pneumoniae causes the purple urine bag syndrome. J Clin Microbiol 1988;26:21526.

4. Keenan CR, Thompson GR 3rd. Purple urine bag syndrome. J Gen In- tern Med 2011;26:1506.

5. Su HK, Lee FN, Chen BA, Chen CC. Purple urine bag syndrome. Emerg Med J 2010;27:714.

6. Chung SD, Liao CH, Sun HD. Purple urine bag syndrome with acidic urine. Int J Infect Dis 2008;12:526-7.

7. Zeier MG, Lee KG, Tan CS. An elderly nursing home resident with unusual urine bag discoloration. NDT Plus 2011;4:445-6. 